\title{
Implantação de um painel de comunicação para equipe multiprofissional no hospital de ensino na cidade de São Paulo
}

Autores:

Adriana Sousa Giovannetti

Edmilson Lorenzoni

Elena Bohomol

Jéssica Aparecida Cardoso 


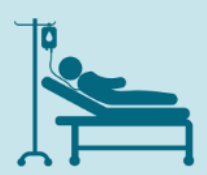

Internação 33.526

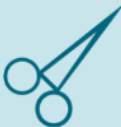

Cirurgias 15.744

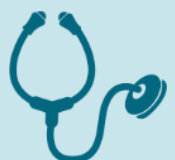

Ambulatório 479.342

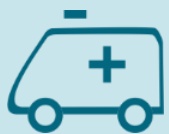

Pronto-socorro 263.086

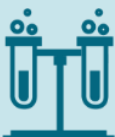

Exames SADT 3.371.620

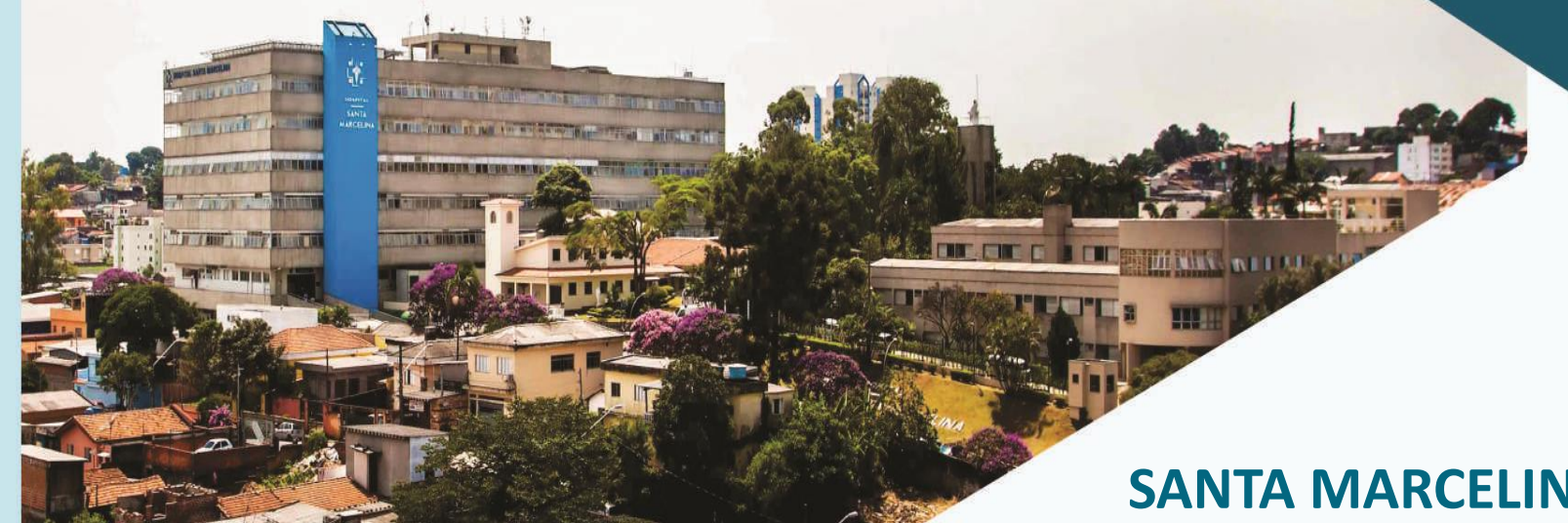

Início das Atividades Itaquera | São Paulo - SP

HOSPITAL SANTA MARCELINA ITAQUERA

Número de leitos 726 Número de leitos UTI 111
Hospital Filantrópico

Referência em Alta Complexidade para Zona Leste

Referência em Ensino e Pesquisa 


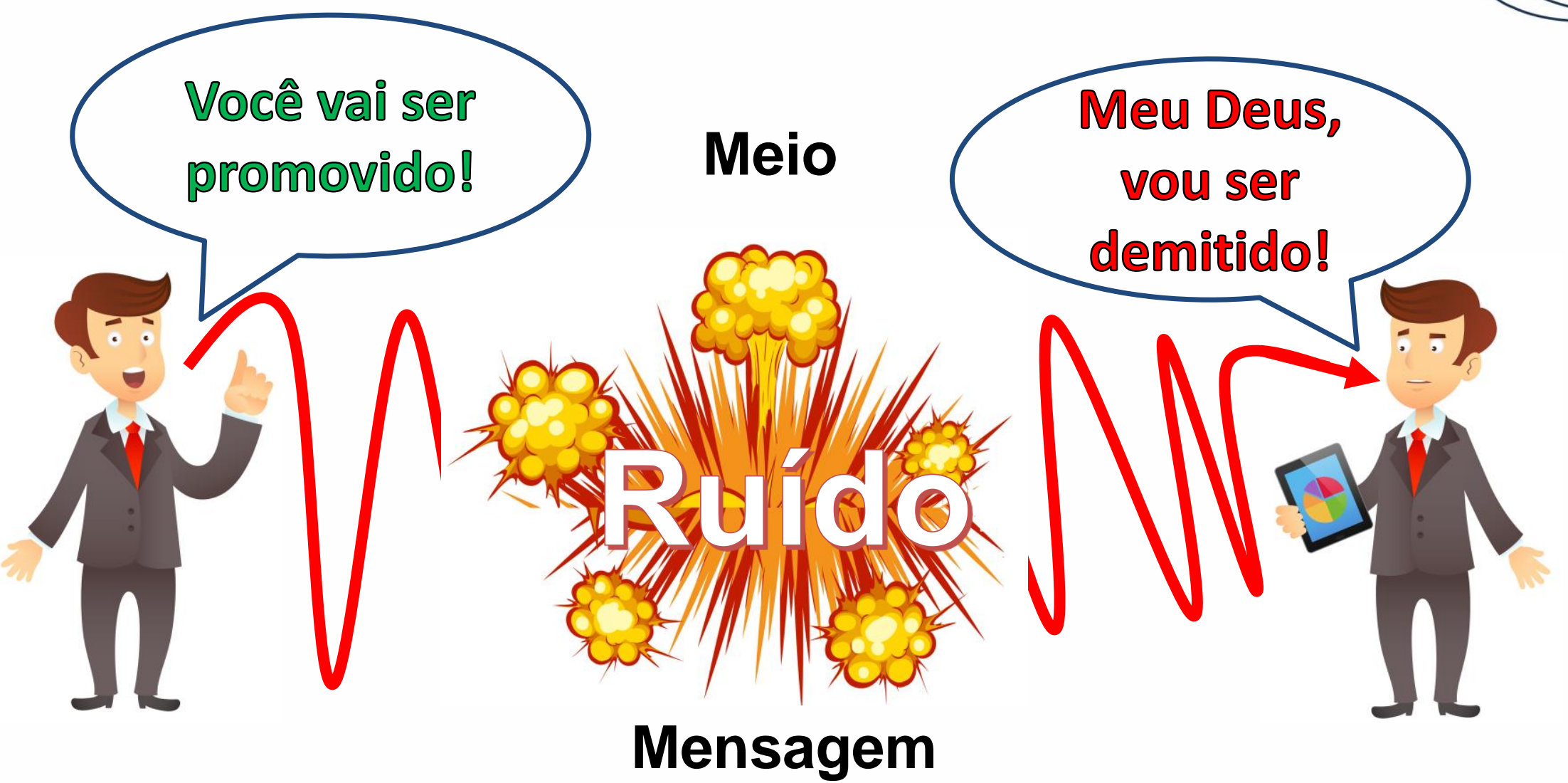

CORIOLANO-MARINUS, Maria Wanderleya de Lavor et al . Comunicação nas práticas em saúde: revisão integrativa da literatura. Saúde soc., São Paulo, v. 23, n. 4, p. 1356-1369, Dec. 2014 
Jejum Prolongado²

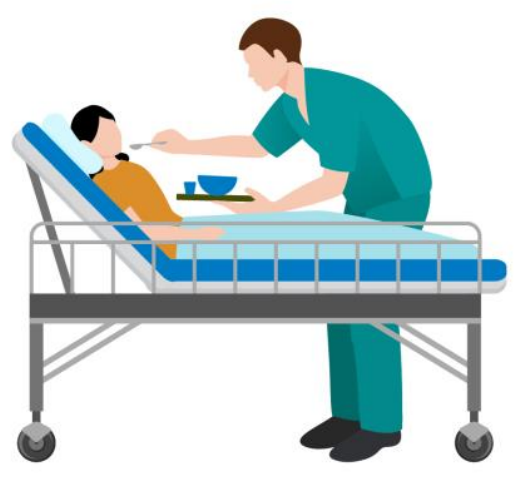

5

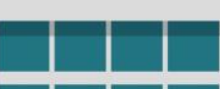

$\square$

-

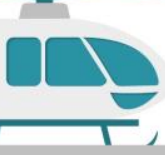

- Emergência Cirúrgica
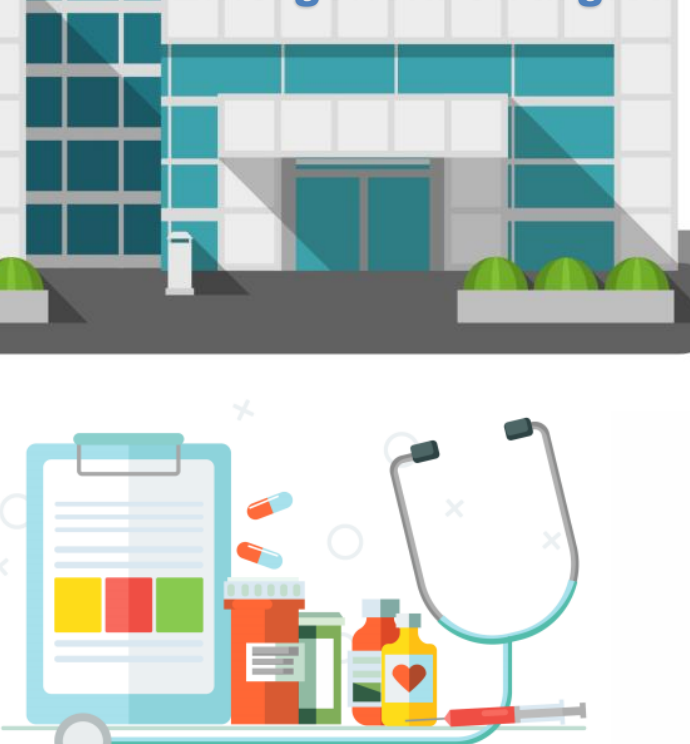

Falta de Prescrição médica ${ }^{4}$

Falha na Passagem das informações ${ }^{3}$ 


\section{Objetivo:}

Implantação de uma intervenção tecnológica, denominada

Painel Assistencial, como forma de monitorar a comunicação entre os profissionais na transição do cuidado.

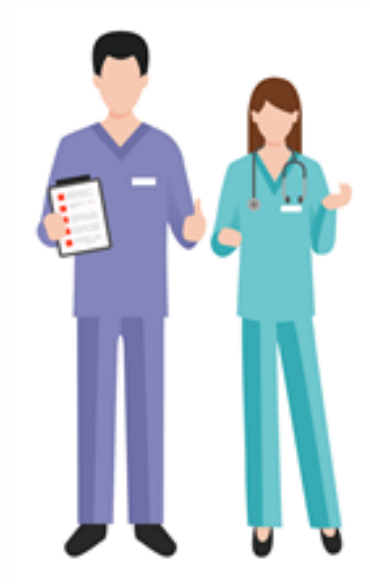




\section{Método:}

- Unidades de internações;

- Reuniões com as equipes multiprofissionais e elencadas as informações relevantes dos pacientes em tempo real;

- Extração automática do banco de dados, baseado no prontuário informatizado;

- Utilizados monitores, expostos nos postos de enfermagem das unidades;

- Treinamento da equipe para o reconhecimentos dos símbolos;

- Acesso individualizado com login e senha na rede interna do hospital. 


\section{Painel Assistencial}

HOSPITAL

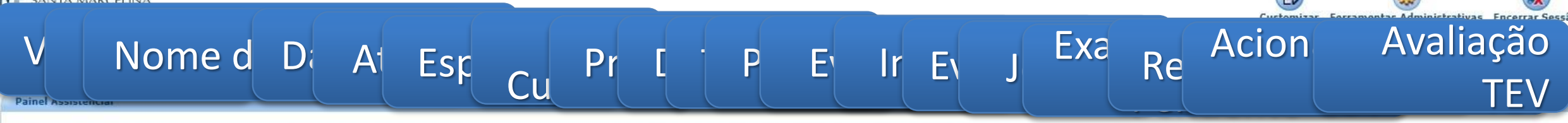

Vis Leito Paciente
Aco

${ }_{03}^{13505 .}$ Controle de Qualidade

13507.
01

2. ${ }_{03}^{13508-}$ Controle de Qualidade $\quad \begin{array}{ll}07 / 12111972 & 15210378 \\ 453 n 05 & \end{array}$

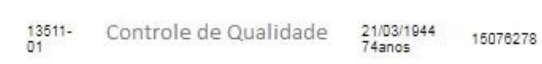

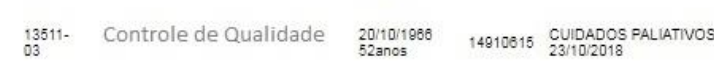

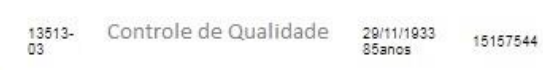

ก. ${ }_{02}^{13515-}$ Controle de Qualidade $\quad \begin{array}{ll}21104 / 1957 & 15206330 \\ 61 \text { anos } & \end{array}$

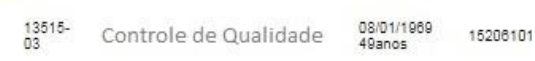

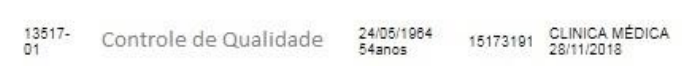

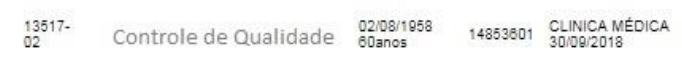

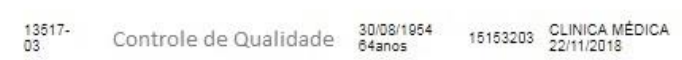

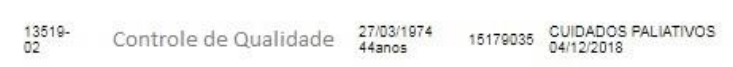

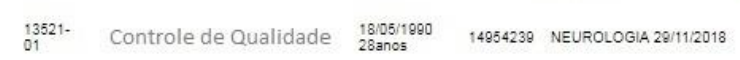

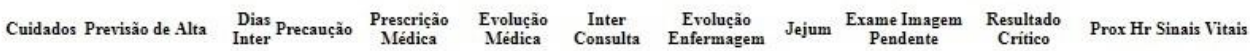
20 $10 / 12 / 2018 \quad 13 \quad \odot$ $20 / 12 / 2018 \quad 27 \quad \&$ $10 / 12 / 2018 \quad 4$ $29 / 10 / 2018 \quad 36 \quad ?$

De. $10 / 11 / 2018 \quad 74$ sem
previsao 16 $30 / 11 / 2018 \quad 4$ $17 / 12 / 2018 \quad 5$

$03 / 12 / 2018 \quad 12$

$30 / 11 / 2018 \quad 87$ $30 / 11 / 18 \quad 18$

07/12/2018 11

$15 / 12 / 2018 \quad 64 \& \overbrace{2}$
(1)

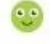

$\odot$

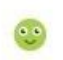

(2) (11)

(1)

$\odot 1$

(1)

()

(1)

2

$\because 2$

(1)

$\odot$

$\therefore$

(1)

(2)

$\because 2$

(2)

$\odot$

(1)
04/12/2018

$20: 30 \odot(0)$

1. $04 / 12 / 2018$

$19: 00 \otimes(2)$

$04 / 12 / 2018$

22:00

$04 / 12 / 2018$

$22: 30$

04/12/2018

$23: 21$

$04 / 12 / 2018$

20:00®(1)

$04 / 12 / 2018$

$20: 51$ (0)

04/12/2018

23:30

$04 / 12 / 2018$

22:30

$19: 00$ (2)

$04 / 12 / 2018$

22:00

$04 / 12 / 2018$

$18: 46 \ominus(2)$

$04 / 12 / 2018$

$18: 47$ (2)
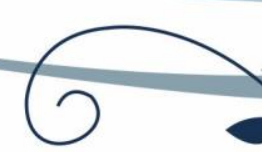

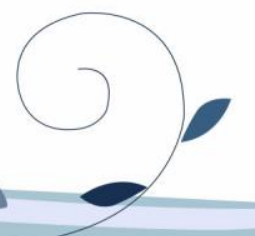

$841201835,9 \quad 2$

7910016

$1031201836,8 \quad 0 \quad 1$

$8911018 \quad 36 \quad 0 \quad 0$

$1091301937,2 \quad 23$

$58701837,2 \quad 3$

$1381101936,6 \quad 14$

$961201734,5 \quad 0 \quad 3$

$1031101835,9 \quad 0 \quad 1$

$6511017 \quad 36 \quad 0$

$741001835,9 \quad 0 \quad 2$

$8512017 \quad 36 \quad 0 \quad 2$

$851201836,5 \quad 0 \quad 0$

(1)

TEV

LUY HANTALAL MARCELINA 


\section{Resultados:}

\section{Diminuição de eventos adversos}

relacionados a atrasos de medicamentos

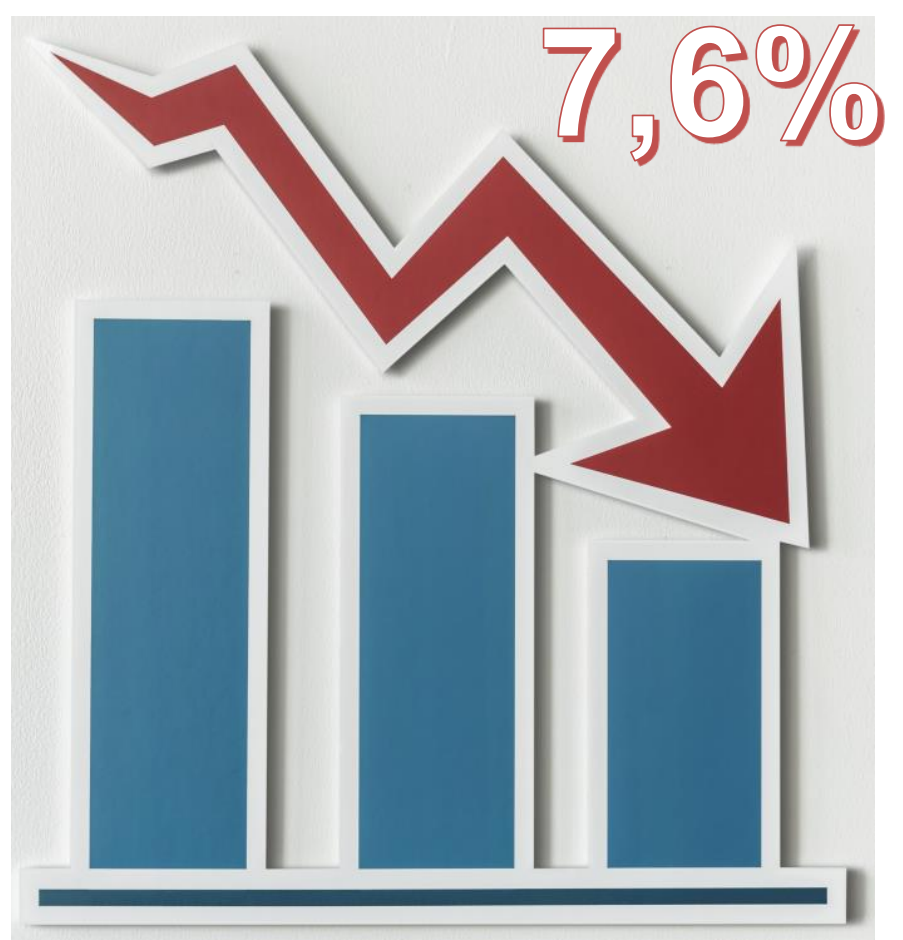




\section{Conclusão:}

- A implantação do painel assistencial foi importante para auxiliar nos processos assistenciais;

- Nortear as equipes multiprofissionais evitando perdas de informações;

- Permitir que os gestores e as equipes monitorem a assistência e a adesão aos protocolos em tempo real. 


\section{Vamos fortalecer as}

barreiras de comunicação!

E prestar um cuidado com mais eficiência e segurança.

\section{Obrigada!}




\section{Referências:}

1. Coriolano-Marinus, Maria Wanderleya de Lavor et al . Comunicação nas práticas em saúde: revisão integrativa da literatura. Saude soc., São Paulo , v. 23, n. 4, p. 1356-1369, Dec. 2014

2. Roque Keroulay Estebanez, Silva Andrea Rodrigues Gomes da, et al. Fatores de risco associados à hipoglicemia e análise de eventos adversos em uma terapia intensiva. Texto contexto - enferm. [Internet]. 2018

3. Nogueira, Jane Walkiria da Silva; Rodrigues, Maria Cristina Soares. Comunicação efetiva no trabalho em equipe em saúde: desafio para a segurança do paciente. Cogitare enferm;20(3): 636-640, Jul-Set. 2015.

4. COSTA, Diovane Ghignatti da et al . Análise do preparo e administração de medicamentos no contexto hospitalar com base no pensamento Lean. Esc. Anna Nery, Rio de Janeiro, v. 22, n. 4, e20170402, 2018. 